\title{
Evaluation of Diffusion-Driven Material Property Profiles Using Three-Wavelength Interdigital Sensor
}

\author{
A. V. Mamishev \\ Department of Electrical Engineering \\ University of Washington, Seattle, WA \\ Y. Du, J. H. Bau, B. C. Lesieutre and M. Zahn \\ . Massachusetts Institute of Technology \\ Department of Electrical Engineering and Computer Science \\ Laboratory for Electromagnetic and Electronic Systems \\ Cambridge, MA USA
}

\begin{abstract}
The response of a three-wavelength interdigital dielectrometry sensor to the moisture diffusion process in oil-impregnated transformer pressboard has been simulated using an empirical relationship between the moisture concentration and the dielectric properties of the pressboard. A benchmark test of the moisture diffusion process has been developed with the purpose of comparing alternative parameter estimation algorithms used in $\omega-k$ (frequency wavenumber) dielectrometry. The results of simulations highlight characteristic features of the multi-wavelength sensor response, such as the sensor response delay from the start of the transient moisture diffusion process as a function of sensor wavelength, the influence of moisture boundary conditions, and the relation between the signal magnitude and variations of dielectric properties. One of the parameter estimation algorithms, linear calibrated admittancebased estimation (LCABE), has been applied to both simulated and measured data. Adequate performance of the LCABE approach in the absence of strong discontinuities of dielectric properties in the electric field penetration region is demonstrated and contrasted to an electrically shielded region case, in which the signal response becomes nonlinear. The proposed approach offers significant potential for the measurement of diffusion processes in various dielectrics, especially for cases with highly irregular geometry or material structure. Measurement results from moisture diffusion process monitoring are included. Parameter estimation of measurement results with LCABE confirm its applicability to the monitoring of moisture dynamics in transformer pressboard.
\end{abstract}

\section{INTRODUCTION}

TNTERDIGITAL sensors are used for a number of research and commercial applications to measure material properties $[1,2]$, control manufacturing processes $[3,4]$, monitor chemical and physical changes of fluid and solid dielectrics $[5,6]$, etc. In many cases, the interpretation of the sensor response depends on simple calibration procedures, yet in other cases, it requires sophisticated signal processing algorithms [7] and a deep understanding of the physics and chemistry of the dynamic processes that are being monitored [8].

Interdigital dielectrometry is a subset of interdigital electrode sensor applications that allows the direct measurement of dielectric properties of insulating and semi-insulating materials from one side [9-11]. The basic idea is to apply a spatially periodic electric potential to the surface of the material under test. Since the changes in the dielectric properties usually are induced by the changes in various physical, chemical, or structural properties of materials, the dielectrometry measurements provide effective means for indirect, non-destructive evaluation and monitoring of vital parameters in a variety of industrial and scientific applications $[12,13]$.

The simplest experimental setup contains a single stationary interdigital sensor in contact with the studied material. In this case, one can only determine dielectric properties of a single homogeneous layer of known thickness, or alternatively, one can determine the thickness of that layer if the dielectric properties are known in advance. The integral 
relationship between the material dielectric properties and the interelectrode admittance can be viewed as an equation with unknowns as dielectric properties on one side and measured or calculated terminal characteristics on the other side.

In order to measure a larger number of unknown parameters, additional equations must be supplied to parameter estimation algorithms. These equations come from the auxiliary instrumentation, physical and numerical models of monitored processes (e.g. diffusion process), and finally, from additional interdigital electrode pairs of different periodicities. For example, the moisture concentration in the ambient fluid may be obtained from an independent commercial sensor and used to better estimate the concentration of moisture in the solid insulation.

The art and science of designing sensors with multiple interdigital electrode pairs and processing the output signals to gain knowledge about the material under test is the subject of interdigital frequency wavenumber $(\omega-k)$ dielectrometry. The penetration depth of the fringing electric fields above the interdigital electrodes is proportional to the spacing between the centerlines of the sensing and the driven fingers and is independent of frequency. Figure 1 illustrates the idea of multiple penetration depths for a three-wavelength sensor. The variation of the material properties across the thickness of the material under test in the vertical direction can be found by simultaneously solving three complex equations describing this experimental arrangement assuming the layers are homogeneous in the horizontal direction.

Multi-wavelength interdigital dielectrometry, also known as frequency wavenumber $(\omega-k)$ dielectrometry, has been under development for $c a$. two decades. An overview of important concepts related to this technology is available $[1,14-20]$.

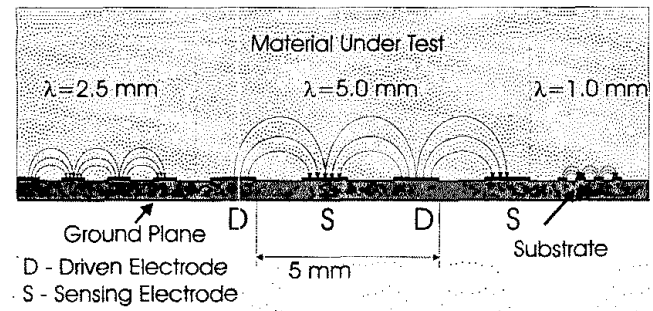

Figure 1. A conceptual view of multi-wavelength dielectrometry showing that the fringing electric field penetration depth is proportional to the fundamental electrode periodicity $\lambda$.

The interdigital frequency-wavenumber dielectrometry methodology is based on electrical excitation of several sets of interdigitated electrodes with a sinusoidal voltage frequency sweep [21]. The combination of signals produced by variation of the spatial period of the interdigital comb electrodes with the variation of electrical excitation frequency provides information about the spatial profiles of dielectric properties of the material under test. The task of interpreting signals is fulfilled by parameter estimation algorithms, whose complexity, precision, accuracy, and reliability vary considerably depending on the experimental conditions and available computation time.

\section{MOTIVATION}

An important application of this technology that is currently under investigation is the ability to study moisture dynamics in power transformer insulation materials in order to prevent failures due to free water formation, excessive losses, and dielectric breakdown induced by flow electrification $[9,13,22,23]$.

Abrupt changes in the operating temperature of large power transformers result in a redistribution of moisture between the transformer oil and pressboard and may lead to transformer failure. Possibilities include the formation of free water in oil, excessive drying of the pressboard-oil interface, accelerated aging, and mechanical degradation of solid transformer insulation.

Dielectric properties of transformer insulation vary with the presence of contaminant materials and additives. Since moisture is always present, it plays an important role in evaluating the operational characteristics of a power transformer. Transformer pressboard, a highly hydrophilic fibrous material, is strongly affected by moisture [24-26], which also makes it a good candidate for monitoring through dielectrometry measurements $[27,28]$. Somewhat paralleling corrosion processes in metals, moisture accelerates pressboard insulation aging due to thermal, mechanical, and electrical degradation of the cellulose base [29-31]. It has been observed that the practical lifetime of cellulose insulation is inversely proportional to its average moisture content [32]. An extensive review of the properties of transformer pressboard, and related measurement, modeling, and application issues is given in [33, 34].

The preliminary measurements of the three-wavelength sensor response to the molecular diffusion process were presented [35]. The goal of this study is to continue the development of parameter estimation algorithms to measure the spatial profiles of dynamically varying moisture concentration across the thickness of transformer pressboard in a controlled environment.

In this paper, the moisture profiles are calculated assuming a nearly linear relationship between the change in sensor transcapacitance and the average amount of moisture in the adjacent pressboard volume [36]. The linearity assumption and terminal circuit parameters are widely used in many designs of relative humidity [37-39], gas [40], chemical [41], and biological $[42,43]$ interdigital sensors. From the true physical representation perspective, this approach is inferior to the full-scale model-based inverse problem algorithms that use electric field modeling to relate spatially varying material properties to lumped circuit elements and electrical network terminal parameters of the sensor. However, it offers several advantages, including simplicity, stability of parameter estimation, speed, and ability to determine moisture profiles in cases of ill-defined geometry and poor surface contact.

\section{THREE-WAVELENGTH SENSOR}

The top view of the sensor used in our recent moisture dynamics studies is shown in Figure 2. It consists of three sets of topologically identical interdigital electrode pairs etched on a common flexible highly hydrophobic Teflon ${ }^{\mathrm{TM}}$ substrate, which is $254 \mu \mathrm{m}$ thick and has a relative dielectric permittivity $\varepsilon_{r}=2.1$. Each sensing comb contains five 
$50 \mathrm{~mm}$ long fingers. Two additional fingers on each side are held at a guard potential equal to the sensing voltage, to approximate the effect of infinite periodicity in the $x$ direction [20]. The gray shaded area indicates guard voltage backplanes on the reverse side of the substrate. The transconductance and transcapacitance between comb electrodes is measured by driving them with a sinusoidal voltage difference. A halfwavelength two-dimensional representation of interdigital electrodes with a superimposed equivalent lumped element circuit is shown in Figure 3.

In our normal practice, the frequency of the driving voltage signal varies between $0.005 \mathrm{~Hz}$ and $10 \mathrm{kHz}$ with 10 logarithmically spaced frequencies per decade. Such a frequency sweep takes $\leqslant 2 \mathrm{~h}$. In order to monitor the diffusion process with sufficient resolution in time, only selected frequency data points were measured and recorded in this experiment. Attention in the following Sections is concentrated on the $1 \mathrm{~Hz}$ frequency measurements. Measurements at other frequencies produce similar results in terms of moisture distribution and provide additional confidence in the proposed approach. High frequency measurements most accurately determine the transcapacitance $C_{12}$ while low frequency measurements most accurately determine the transconductance $G_{12}$. For the most accurate measurements to determine both the transcapacitance and transconductance, the optimum frequency is on the order of $f=G_{12} /\left(2 \pi C_{12}\right)$.

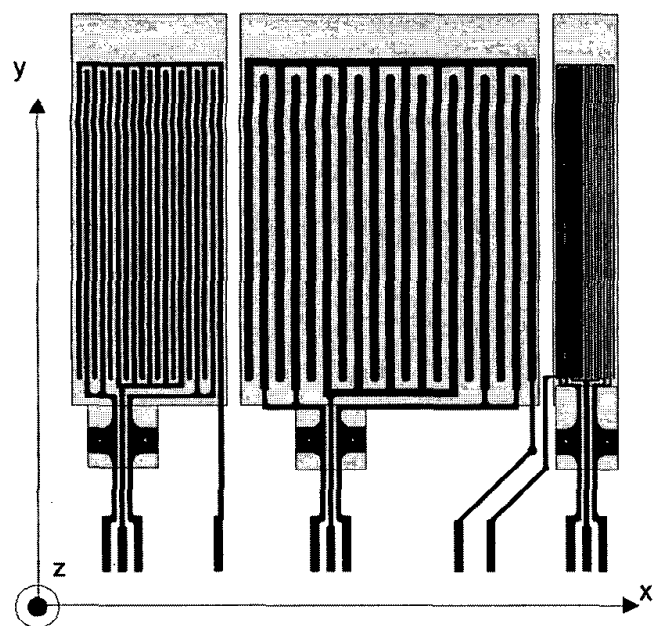

Figure 2. The three-wavelength interdigital sensor with spatial periodicities of $2.5,5.0$, and $1.0 \mathrm{~mm}$.

\section{SEQUENCE OF COMPUTATIONS}

Whenever a new algorithm to solve the inverse problem is being developed, it should be tested first on synthetic data, free of experimental noise and statistical measurement errors. In order to evaluate the performance of the linear calibrated admittance-based estimation (LCABE), the admittance output for various frequencies of the three-wavelength sensor as a function of time during the moisture diffusion process has

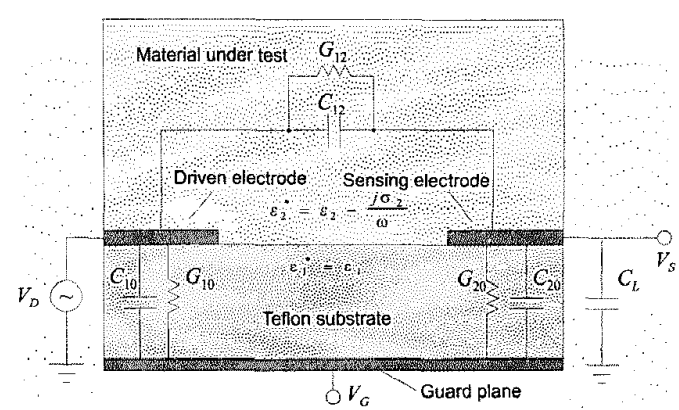

Figure 3. Half-wavelength representation with superimposed equivalent lumped element circuit.

been simulated using typical values of physical parameters of transformer pressboard. Then, admittance values were supplied to LCABE as the input for the inverse problem to determine moisture profile as a function of time. The sequence of this study is outlined below:

1. Simulate the moisture diffusion process by numerically solving the governing moisture diffusion equation with a finite-difference code.

2. Convert spatial moisture profiles for each measurement frequency at several distinct moments of time to the profiles of dielectric propertics, using measured relationships from [18].

3. Discretize the calculated dielectric property profiles with a spatial resolution significantly higher than a three-layer approximation suggested by the penetration depths of the three-wavelength sensor in Figure 4.

4. Using the finite element simulation (Maxwell ${ }^{\mathrm{TM}}$ by Ansoft Corp.), generate a table of values of transcapacitance $C_{12}$ and transconductance $G_{12}$ for each property profile and each wavelength of the sensor.

5. For each moment of time that has an associated property profile, generate a set of six input variables ( $G_{12}$ and $C_{12}$ for each wavelength of the three-wavelength sensor).

6. Use time curves of $C_{12}$ and/or $G_{12}$ to extrapolate the moisture profile at each selected moment of time using the LCABE algorithm.

7. Convert the stair-step moisture profiles to either piecewise-linear or smooth profiles, because the nature of the diffusion process requires that property profiles should not have discontinuities across layer boundaries.

8. Compare the results of the inverse problem solution with the original simulated data of moisture dynamics.

\section{FORWARD PROBLEM}

The forward problem simulation of calculating the equivalent circuit parameters given all material properties includes steps 1 through 5. Several alternative methods could have been used to implement each step. For example, the diffusion equation could have been solved either numerically or analytically. We chose to use a numerical solution to the diffusion equation with constant diffusion coefficient, because in future work we plan to allow the diffusion coefficient to be a function of moisture concentration, for which analytical solutions are either difficult or not possible. Also, the conversion of moisture profiles into dielectric property profiles depends on empirical data that strongly deviate from the best fit due to a limited spatial resolution of the sensor. However, as long as one is consistent in the assumptions made for the forward 
problem and for the inverse problem, the performance of the inverse algorithm is assessed properly.

\subsection{DIFFUSION PROCESS}

Figure 5 shows spatial profiles of moisture concentration generated with a finite difference simulation program according to a classic onedimensional diffusion equation with a constant diffusion coefficient $D=10^{-11} \mathrm{~m}^{2} / \mathrm{s}$.

$$
\frac{\partial m}{\partial t}=D \frac{\partial^{2} m}{\partial x^{2}}
$$

where $m$ is moisture concentration, $t$ the time, and $x$ is the spatial coordinate in the direction of diffusion. The profiles are shown at 10 distinct moments of time, and the number next to each curve represents time in hours elapsed since the beginning of the diffusion process. The left boundary condition at $x=0$ is that the moisture concentration is at a constant value, chosen to be $10 \%$ throughout the process. This boundary condition corresponds to the case when a relatively small amount of pressboard is placed in a very large oil container or when it is open to the atmosphere and the relative humidity of air does not vary significantly during the experiment. Since the total moisture concentration in a sealed transformer is fixed, a change in moisture level in pressboard also changes the moisture level in oil. However, a constant boundary condition is more desirable for the benchmark problem to insure simplicity and consistency of subsequent analysis. The right boundary condition at $x=1 \mathrm{~mm}$ is the zero flux across the boundary. This condition exists when the pressboard faces an impermeable wall on one side. The sensor attached to the side of pressboard provides this type of an interface.

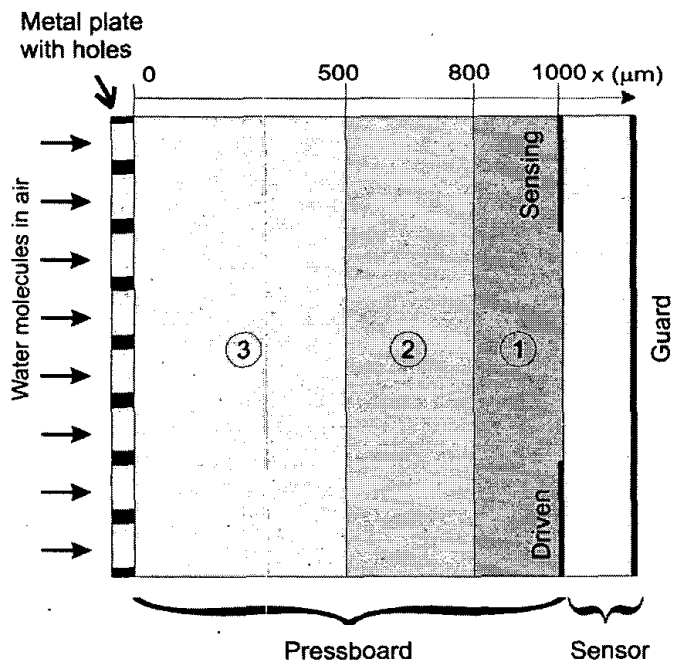

Figure 4. The cross-section of pressboard may be discretized into three layers. For each layer the distance from the sensor to the far interface corresponds to one-fifth of each of the three spatial wavelengths of the sensor.

The diffusion process starts at time zero and continues for $\sim 30 \mathrm{~h}$. After $30 \mathrm{~h}$, a nearly flat pritile is obtained. The sensor should detect

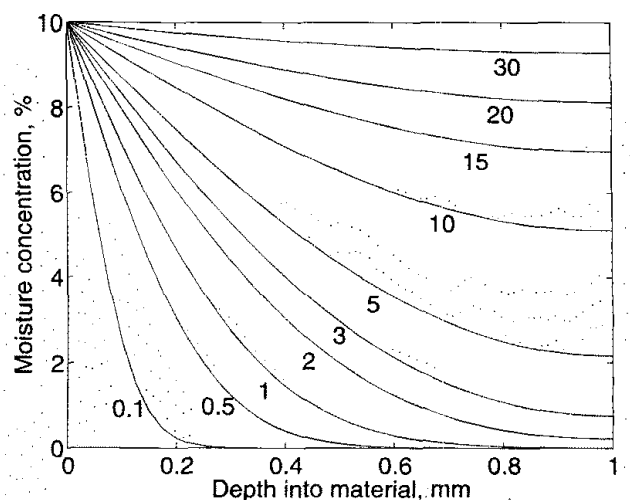

Figure 5. Calculated spatial profiles of moisture concentration across the $1 \mathrm{~mm}$ thickness of pressboard from dielectrometry measurements using $D=10^{-11} \mathrm{~m}^{2} / \mathrm{s}$. Numbers next to the curves correspond to the time (h) at which the profile was calculated.

nearly homogeneous properties across the entire pressboard thickness in the beginning and at the end of the simulated period of time.

Characteristics of the imposed boundary conditions are steep gradients close to the left boundary and almost flat profiles close to the right boundary. Consequently, shorter wavelengths see a nearly homogeneous region, while the fringing fields from the longer wavelengths penetrate through highly inhomogeneous regions. The signal indicating the change of moisture concentration after it was stepped up at the left boundary is expected to be practically the same for the 1 and $2.5 \mathrm{~mm}$ wavelengths that are most sensitive to changes in regions 1 and 2 shown in Figure 4 . The right-side boundary condition $(x=1000 \mu \mathrm{m})$ of no flux across the interface causes the moisture profiles to be fairly uniform. Since the penetration depth of the largest $5 \mathrm{~mm}$ wavelength is approximately equal to the pressboard thickness, the signal delay for this wavelength is essentially zero, while there will be some delay before the moisture diffuses to within the penetration depths for each of the shorter 2.5 and $1 \mathrm{~mm}$ wavelengths.

\subsection{DIELECTROMETRY PROFILES}

In the next step, moisture profiles are converted into profiles of dielectric properties for oil-impregnated pressboard using semi-empirical relationships available from [13]

$$
\begin{aligned}
\varepsilon^{\prime} & =\varepsilon_{\infty}+\varepsilon_{o} 10^{-\gamma\left(f_{T}+c_{m}\right)} 10^{c_{1}}\left(\frac{m}{f}\right)^{-\gamma} \\
\varepsilon^{\prime \prime} & =\varepsilon_{o} 10^{-\gamma\left(f_{T}+c_{m}\right)} 10^{c_{2}}\left(\frac{m}{f}\right)^{-\gamma}
\end{aligned}
$$

The functional form of these relationships originates from the 'universal curve' methodology in [44-46]. The values of empirical coefficients have been obtained by curve fitting of experimental data [18]. The following values of coefficients were used: $c_{1}=-0.7798, c_{2}=-0.4724$, $c_{m}=-0.684, f_{T}=0.6, \gamma=-0.7$. The high frequency relative dielectric permittivity of transformer pressboard is assumed to be $\varepsilon_{\infty}=3$. (The experimental data discussed later has been obtained only for a narrow frequency range $(1 \mathrm{~Hz}$ to $10 \mathrm{~Hz})$. For that reason, it is not possible to 
see how well (2) and (3) obey the Kramers-Kronig relationships which rely on integrals over the entire frequency range from 0 to $\infty$.)

For the parameters analyzed in this problem, the relationship between the moisture and the dielectric properties are not far from linear. Not surprisingly, the time-dependent theoretical profiles of dielectric properties in Figures 6 and 7 look very similar to the moisture profiles in Figures 5. Figure 6 shows profiles of relative dielectric permittivity during the diffusion process. The maximum value of $\varepsilon_{r}=3.72$ is determined from (2) by the left boundary condition for moisture concentration $m=10 \%$. (This simulation excludes low frequency dispersion effects due to an electrochemical double layer, when the estimated values of dielectric permittivity are much higher than the high frequency permittivity.)

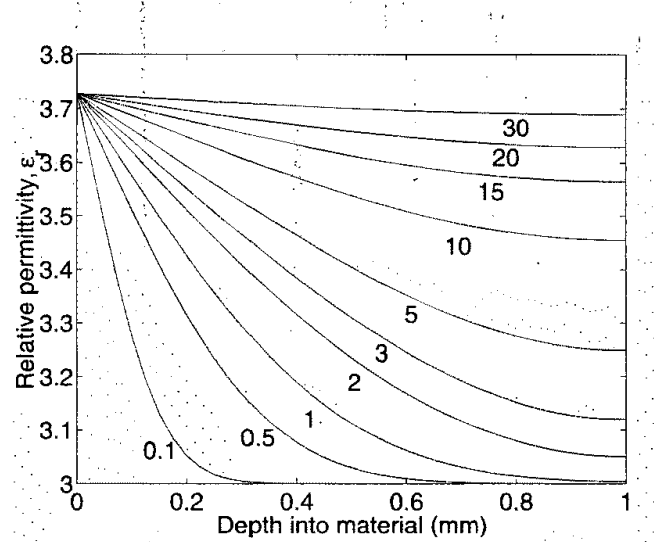

Figure 6. Calculated spatial profiles of relative dielectric permittivity $\varepsilon_{r}=\varepsilon^{\prime} / \varepsilon_{o}$ at $1 \mathrm{~Hz}$ across the thickness of pressboard using (2) and Figure 5. Numbers next to the curves correspond to the time (h) for which the profile was measured.

Spatial profiles of the imaginary part of the complex dielectric permittivity (loss factor) obtained using (3) are very similar in shape to those of the real part of complex dielectric permittivity, but they span a relatively larger range of values. For that reason, and also since the minimum value of $\varepsilon^{\prime \prime}$ is zero, the loss tangent $\tan \delta=\sigma /\left(\omega \varepsilon^{\prime}\right)$ profiles (not shown here) are also similar in shape. Assuming that the measurements are done at $1 \mathrm{~Hz}$, the loss factor profiles $\left(\varepsilon^{\prime \prime}=\sigma / \omega\right)$ can be recalculated readily into conductivity profiles by multiplying them by $\omega=2 \pi$. The conductivity profiles are presented separately in Figure 7 because they are related directly to the time variation of the transconductance $G_{12}$ discussed later.

\subsection{STAIR-STEP APPROXIMATION}

The sensor response to gradual changes of moisture concentration has been simulated using commercial finite-element software Maxwell. The cross-section of pressboard has been broken into 20 equally thick layers, each with specified distinct values of relative permittivity and conductivity, so that for each moment of time this spatial profile would be approximated in a fashion shown in Figure 8.

The broken curve shows the original profile of relative dielectric permittivity of pressboard at $t=1 \mathrm{~h}$, and the solid line shows a corre-

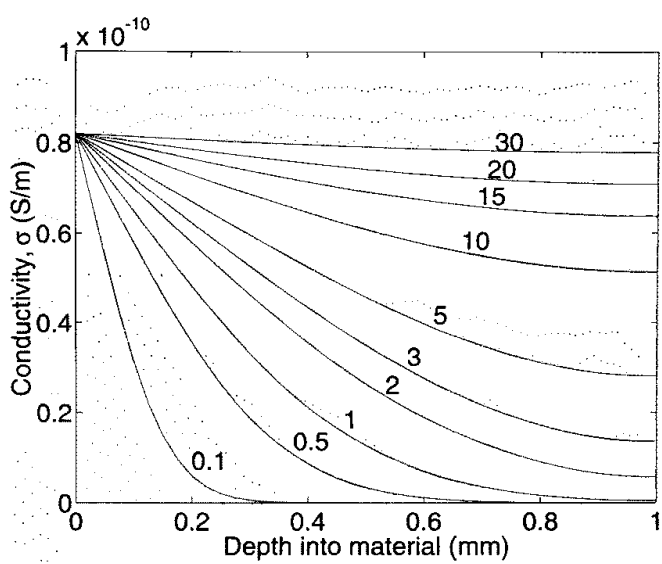

Figure 7. Calculated spatial profiles of conductivity $\left(\sigma=\omega \varepsilon^{\prime \prime}\right)$ across the thickness of pressboard using (3) and Figure 5 with $\omega=2 \pi$. Numbers next to the curves correspond to the time (h) for which the profile was calculated.

sponding 20 layer stair-step approximation. This representation is significantly more accurate than a three-step representation available from the three-wavelength sensor. The computational error introduced by the stair-step representation is well within the the maximum accuracy available from the three-wavelength sensor.

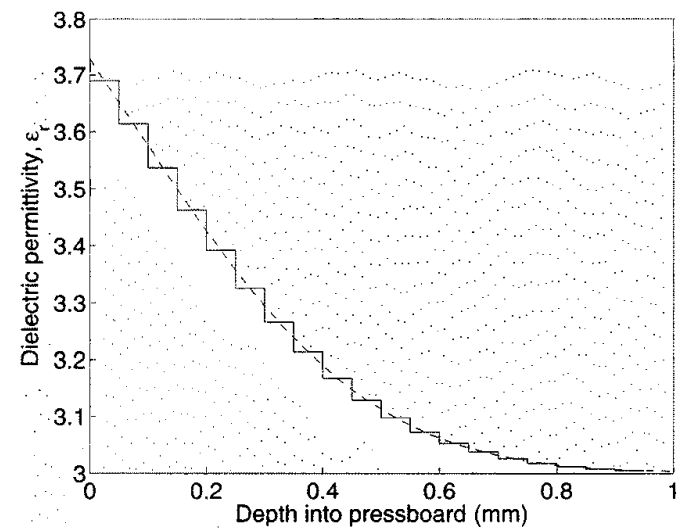

Figure 8. Twenty-layer stair-step representation of a continuous spatial profile of relative dielectric permittivity of pressboard at $t=1 \mathrm{~h}$.

Figure 9 shows the finite element mesh generated by Maxwell for the cross-section of the $2.5 \mathrm{~mm}$ wavelength sensor. The density of the mesh is higher at the electrode edges, in the regions of significant electric field enhancement. The location and shape of triangles in the upper part of the pressboard cross-section reflects the layered representation illustrated in Figure 8. For the purpose of this simulation, the thin layer between the driven and sensing electrodes whose thickness is equal to the electrode height $(14 \mu \mathrm{m})$ is assigned the same properties as the insulation layer directly above it. Other simulations could allow this cavity to have dielectric properties of oil for oil-impregnated pressboard or air for oil-free pressboard. 


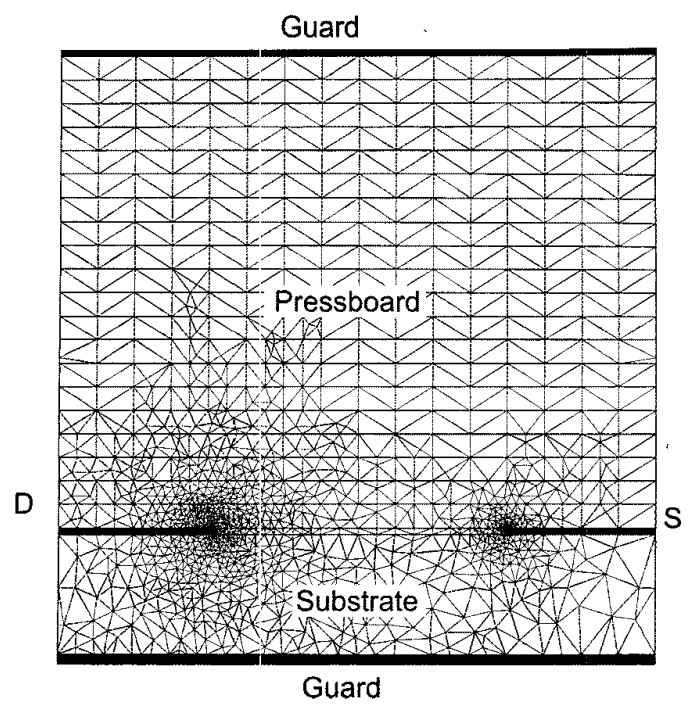

Figure 9. Finite-element mesh for the $2.5 \mathrm{~mm}$ wavelength electrode cross-section with the guard electrodes at the same potential as the sensing electrode and where spatially varying properties of transformer pressboard are modeled through a twenty layer representation.

\subsection{RESULTS OF NUMERICAL SIMULATION}

The admittance between the driven and sensing electrodes for each spatial wavelength is calculated for each moment of time indicated in Figure 5. Two cases are considered, corresponding to our experimental setups. The first case assumes that the region above the pressboard is of semi-infinite extent and has dielectric properties of Teflon, $\varepsilon_{r}=2.1$. The second case assumes that a guard plane at the same potential as the sensing electrode is positioned at the top of the pressboard (for the sensor and pressboard orientation shown in Figure 9). One experimental setup uses a guarded perforated steel plate to provide mechanical support while allowing access of the external fluid (usually air or transformer oil) to pressboard. Alternatively, a perforated Teflon plate has been used. A third case, not analyzed here, is direct access of the external fluid to the pressboard-fluid interface, without a perforated plate. In this case, the signal dynamics should be similar to that in case one, because the Teflon plate is closer in dielectric properties to the dielectric fluid than to a metal plate.

A major distinction exists for the conductors and insulators positioned at the top of the $1 \mathrm{mrn}$ thick pressboard cross-section. The presence of a conducting sheet significantly alters the distribution of fringing electric fields throughout the thickness of pressboard, especially in the upper region. This, in turn, affects the values of transcapacitance $C_{12}$ and transconductance $G_{12}$ between the driven and sensing electrodes. Table 1 shows values of transconductance $G_{12}$ and transcapacitance $C_{12}$ computed for all three wavelengths, assuming a $50 \%$ metalization ratio (equal width drive and sense electrodes), $1 \mathrm{~m}$ long meander length, and a top insulating layer of infinite thickness with a relative dielectric permittivity $\varepsilon_{r}=2.1$ (Teflon). Table 2 shows the values
Table 1. Nominal values of transconductance $G_{12}$ and transcapacitance $C_{12}$ for all three wavelengths calculated for 11 distinct moments of time during the diffusion process assuming a top insulating layer of semi-infinite extent with $\varepsilon_{r}=2.1$ (Teflon).

\begin{tabular}{|c|r|r|r|r|r|r|}
\hline \hline Time & \multicolumn{2}{|c|}{$\lambda=1.0 \mathrm{~mm}$} & \multicolumn{2}{c|}{$\lambda=2.5 \mathrm{~mm}$} & \multicolumn{2}{c|}{$\lambda=5.0 \mathrm{~mm}$} \\
\cline { 2 - 7 } $\mathrm{h}$ & $G_{12} \mathrm{pS}$ & $C_{12} \mathrm{pF}$ & $G_{12} \mathrm{pS}$ & $C_{12} \mathrm{pF}$ & $G_{12} \mathrm{pS}$ & $C_{12} \mathrm{pF}$ \\
\hline 0 & 0.0000 & 16.5209 & 0.0000 & 10.3963 & 0.0000 & 7.0421 \\
0.1 & -0.0107 & 16.5195 & 0.1381 & 10.4090 & 0.6713 & 7.1047 \\
0.5 & 0.0011 & 16.5197 & 0.6135 & 10.4520 & 1.8974 & 7.2173 \\
1 & 0.4254 & 16.5529 & 1.6345 & 10.5389 & 3.2680 & 7.3356 \\
2 & 3.5564 & 16.7989 & 4.8608 & 10.7960 & 5.9749 & 7.5466 \\
3 & 7.8792 & 17.1368 & 8.4096 & 11.0657 & 8.4174 & 7.7200 \\
5 & 15.7712 & 17.7502 & 14.4998 & 11.5137 & 12.3269 & 7.9809 \\
10 & 28.3175 & 18.7188 & 24.1318 & 12.2030 & 18.4244 & 8.3686 \\
15 & 35.0905 & 19.2391 & 29.4096 & 12.5739 & 21.8070 & 8.5771 \\
20 & 39.0242 & 19.5407 & 32.5008 & 12.7893 & 23.8041 & 8.6982 \\
30 & 42.8133 & 19.8306 & 35.4947 & 12.9967 & 25.7488 & 8.8146 \\
\hline \hline
\end{tabular}

Table 2. Nominal values of transconductance $G_{12}$ and transcapacitance $C_{12}$ for all three wavelengths calculated for 11 distinct moments of time during the moisture diffusion process assuming a perfectly conducting top layer.

\begin{tabular}{|c|r|r|r|r|r|r|}
\hline \hline \multirow{2}{*}{$\begin{array}{c}\text { Time } \\
\mathrm{h}\end{array}$} & \multicolumn{2}{|c|}{$\lambda=1.0 \mathrm{~mm}$} & \multicolumn{2}{c|}{$\lambda=2.5 \mathrm{~mm}$} & \multicolumn{2}{c|}{$\lambda=5.0 \mathrm{~mm}$} \\
\cline { 2 - 7 } 0 & $G_{12} \mathrm{pS}$ & $C_{12} \mathrm{pF}$ & $G_{12} \mathrm{pS}$ & $C_{12} \mathrm{pF}$ & $G_{12} \mathrm{pS}$ & $C_{12} \mathrm{pF}$ \\
0.1 & 0.0000 & 14.0861 & 0.0000 & 5.6639 & 0.0000 & 1.3092 \\
0.5 & -1.3645 & 13.0140 & -0.9099 & 5.5501 & -0.4919 & 1.2453 \\
1 & -1.5941 & 13.9055 & -2.1000 & 5.4152 & -0.9600 & 1.1869 \\
2 & 0.5577 & 14.0798 & -0.8331 & 5.5293 & -0.5285 & 1.2321 \\
3 & 4.1422 & 14.3718 & 1.1481 & 5.7023 & 0.0800 & 1.2904 \\
5 & 10.9672 & 14.9225 & 4.8925 & 6.0143 & 1.2252 & 1.3886 \\
10 & 21.9644 & 15.7952 & 11.0511 & 6.4958 & 3.1819 & 1.5320 \\
15 & 27.9077 & 16.2604 & 14.4556 & 6.7488 & 4.3084 & 1.6043 \\
20 & 31.3596 & 16.5289 & 16.4557 & 6.8938 & 4.9841 & 1.6448 \\
30 & 34.6853 & 16.7866 & 18.3975 & 7.0324 & 5.6495 & 1.6829 \\
\hline \hline
\end{tabular}

of $G_{12}$ and $C_{12}$ computed for the same case, but with the top insulating layer being replaced by a perfect conductor. Although graphical representation of this data is also included and analyzed below, the response is tabulated because these cases are expected to serve as benchmark tests for alternative parameter estimation algorithms.

To avoid confusion, we should comment on the negative values of the transconductance $G_{12}$ in column 2 of Table 1 and in columns 2, 4, and 6 of Table 2 . This does not violate any physical laws and does not require any special assumptions about the physics or electrochemistry of the process. The negative values of transconductance (as well as transcapacitance) arise from the fact that a multi-electrode distributed system with spatially varying material properties is being represented as the simple lumped element circuit of Figure 3 that does not have the same topology as the sensor. In particular, there is a parallel capacitance and conductance from the interface between drive and sense electrodes to the backplane guard electrodes that at low frequencies can result in negative values of $C_{12}$ and $G_{12}$ in the $\pi$-equivalent circuit. Additional discussion to clarify this issue is available [20].

The simulated change of capacitance with time, from values in Tables 1 and 2 are shown for each wavelength in Figures 10(a) and (b), respectively, without and with a metal top plate. The absolute value of transcapacitance increases as the spatial wavelength decreases for the same total meander length. Also, the absolute value of $C_{12}$ decreases 
with the introduction of the perfect conductor as a top plate. Intuition supports this result: the conductor terminates some of the electric field lines originating at the driven electrode which would otherwise have reached the sensing electrode. So, even though the total capacitive energy stored in the system increases, the capacitive coupling between the driven and sensing electrodes decreases because of redirection of fringing electric field lines.

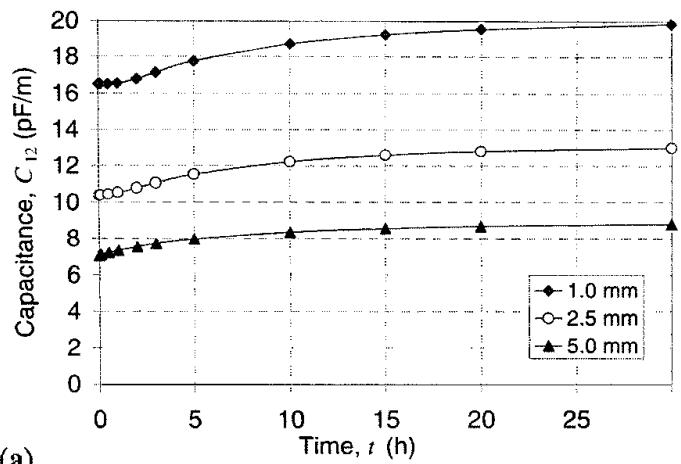

(a)

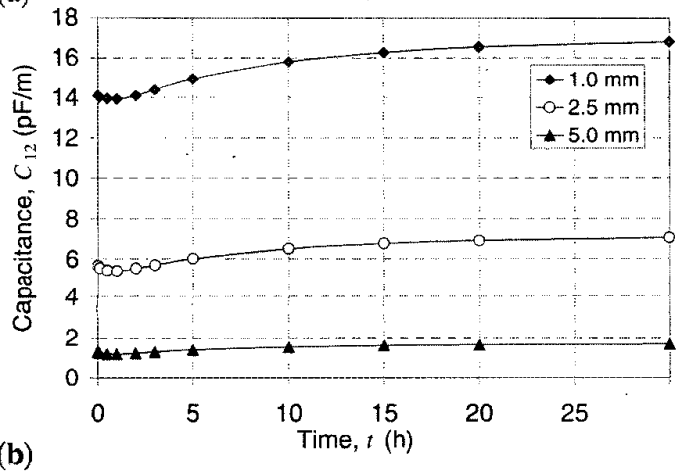

Figure 10. Simulated variation of capacitance per meter during moisture diffusion calculated using the half-cell presentation as shown in Figure 9. (a) without metal top plate, (b) with metal top plate.

For the purposes of employing the LCABE algorithm, the time varying capacitive sensor response should be normalized to common base values, which will be related later to moisture concentration base values. First, consider normalization to the starting value, shown in Figure 11

$$
C_{12 n s}=\frac{C_{12}(t)}{C_{12}(t=0)}
$$

The a priori number at $t=0$ is available because the entire pressboard region is considered uniform before the diffusion process starts. Therefore, one may want to use the starting value of capacitance of each wavelength as the normalization factor. Each curve in Figure 11 was obtained by dividing entries in Figure 10 by the corresponding curve starting value, for example, by $C_{12}=7.0421 \mathrm{pF} / \mathrm{m}$ in Table 1 for the $5.0 \mathrm{~mm}$ wavelength curve in Figure 11(a). This way of plotting emphasizes some salient features of the process dynamics. The relative change of capacitance increases with wavelength, because the ratio of the spatial wavelength to the substrate thickness approximately deter-

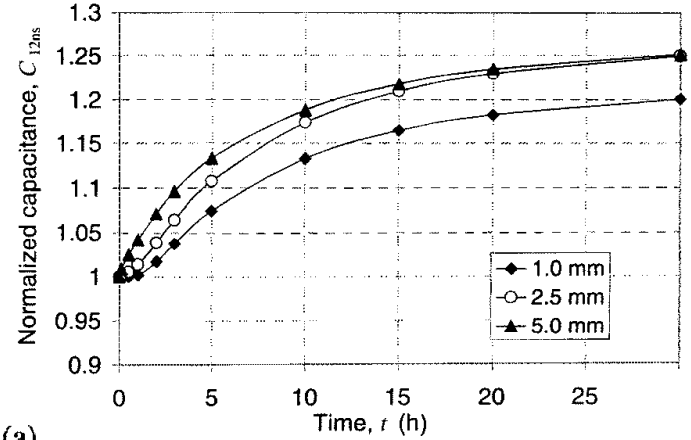

(a)

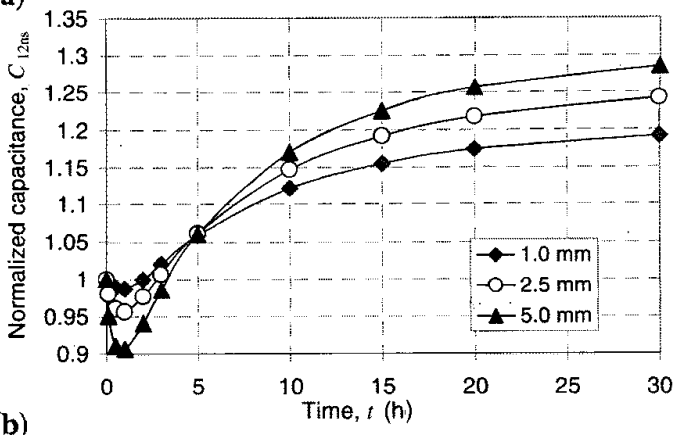

(b)

Figure 11. Change of normalized capacitance $C_{12 n s}$ during the diffusion process with normalization to the starting value for each of the three wavelengths as given in (4). (a) without metal top plate, (b) with metal top plate.

mines what fraction of energy is stored in the pressboard. Since the substrate thickness does not change with the wavelength in this sensor design, the final normalized values of capacitance differ at the end of the diffusion process. The only exceptions are the 5.0 and $2.5 \mathrm{~mm}$ wavelengths in Figure 11(a), whose values are approximately equal at $t=30 \mathrm{~h}$. This happens because a small part of capacitive energy associated with the $5.0 \mathrm{~mm}$ wavelength is stored in the top insulating layer, which reduces the fraction of the electric field lines that go only through the pressboard. In other words, if the thickness of pressboard was higher, the distribution of the final normalized values of capacitance $C_{12 n s}$ in Figure 11(a) would be similar to that observed in Figure 11(b).

Figure 11 illustrates the delay difference with wavelength. In case (a), the $5.0 \mathrm{~mm}$ wavelength reacts to changes in moisture concentration immediately, whereas the $1.0 \mathrm{~mm}$ wavelength goes through the inflection point, experiencing a delay on the order of $0.5 \mathrm{~h}$. In case (b), all wavelengths respond immediately, although with different intensity, because the zero order spatial Fourier harmonic is affected strongly by the large increase in conductivity and permittivity due to the presence of moisture in the upper region of the pressboard cross-section. Another way to look at this is to say that the fringing electric field lines from the drive electrode to the top plate are affected immediately by the increase of conductivity and dielectric permittivity at the top.

Another important feature of the time profiles in Figure 11(b) is the initial decrease of capacitance during the first $3 \mathrm{~h}$ of the diffusion process. It may seem counter-intuitive at first, because the average value of 


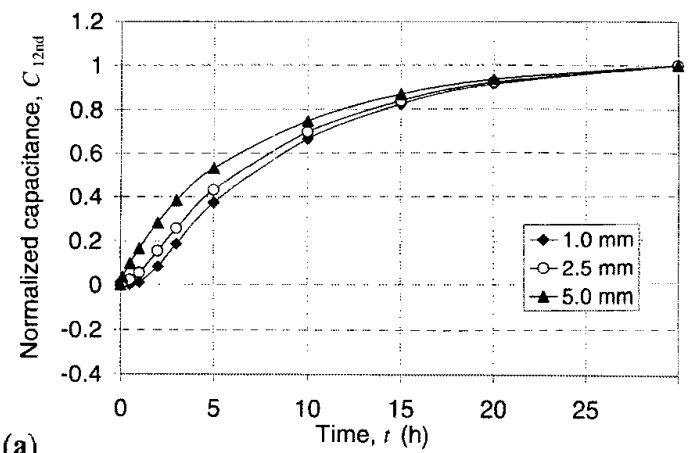

Figure 12. Change of normalized capacitance during the diffusion process with normalization to the difference between the final and the starting values for each of the three wavelengths as given in (5). (a) Without metal top plate, (b) with metal top plate.

the relative dielectric permittivity grows continuously. In this case, the initial reduction of capacitance is due to increasing conductivity in the upper region. Some fringing electric field lines that previously would follow an arc between the driven and sensing electrode now are drawn into the high conductivity region and terminate on the top plate.

Another possible way to normalize the change of capacitance is by using a posteriori information. Figure 12 shows the same signal curve linearly normalized to the difference between the final and the starting value, so that the final value of the normalized signal is equal to 1 and the starting value is equal to 0 :

$$
C_{12 n d}=\frac{C_{12}(t)-C_{12}(t=0)}{C_{12}(t=\infty)-C_{12}(t=0)}
$$

Figure 12(a) exhibits similar features as Figure 11(a). The speed of the response growth increases with the spatial wavelength. At each moment of time, except for the initial and final values, the magnitude of the normalized capacitance $C_{12 n d}$ is the highest for the $5.0 \mathrm{~mm}$ wavelength and the lowest for the $1.0 \mathrm{~mm}$ wavelength. From the perspective of mapping the range of capacitance values to the range of moisture values, the representation in Figure 12(a) is more advantageous than that in Figure 11(a) because it allows direct linear mapping in which the average signals from each region of the pressboard retain their relative relationships. On the other hand, the relative positioning of normalized signal amplitude is reversed in Figure (b) with respect to their counterparts in Figure (a). A direct linear mapping to moisture values would

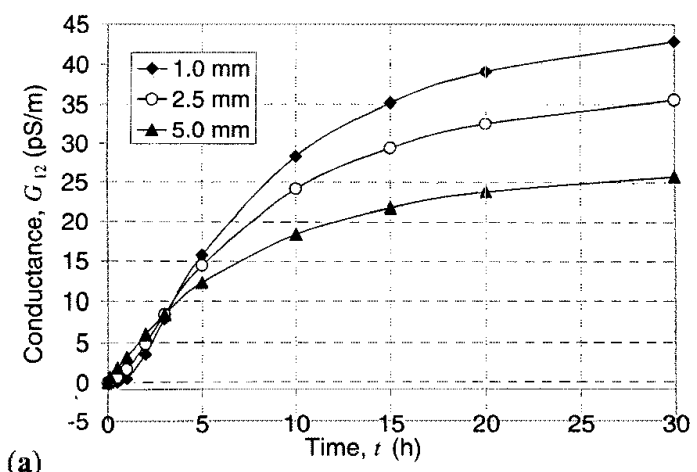

(a)

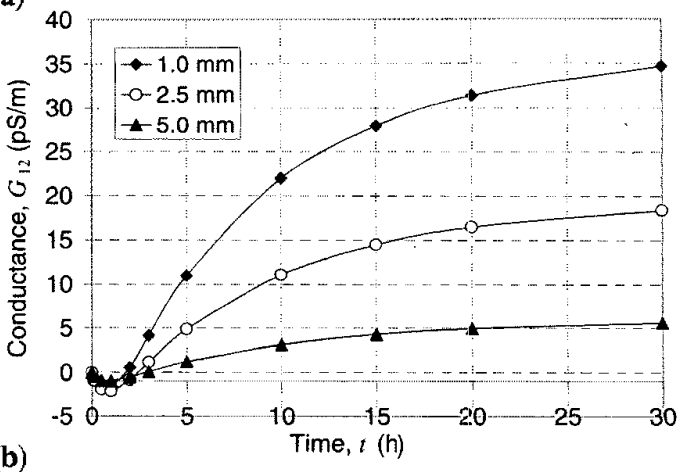

Figure 13. Simulated variation of conductance during moisture diffusion per meter length, calculated using a half-cell representation as shown in Figure 9. (a) Without metal top plate, (b) with metal top plate.

result in non-physical negative moisture concentration values early in the simulation.

Before we turn our attention to mapping of capacitance dynamics to moisture dynamics, we should also review the changes of the interelectrode transconductance during the same diffusion process. It is well known that the local bulk conductivity of transformer pressboard changes by several orders of magnitude during typical moisture concentration transients [13], whereas the real part of the complex dielectric permittivity varies in a comparatively narrow range (at a given frequency). Figure 13 reflects this difference. The initial conductance values are zero, whereas the final values are on the order of $10 \mathrm{pS}$.

The effect of the metal top plate is clearly pronounced. The distribution of the final values of $G_{12}$ is much wider for case (b) in Figure 13. This same effect has been observed in Figure 10 for the capacitance $C_{12}$ values. The intersection of curves in Figure 13(a) at $\sim 3 \mathrm{~h}$ from the beginning of the diffusion process that leads to the reversal of the wavelength sequence in their signal's relative magnitude for each period of time is easy to interpret. Given an infinitely thick homogeneous material, the conductance decreases with the wavelength increase, so when the property distribution becomes nearly homogeneous at time $t=30 \mathrm{~h}$, the conductance increases from the $5.0 \mathrm{~mm}$ wavelength to the $1.0 \mathrm{~mm}$ wavelength. However, in the beginning of the process, when the moisture has only partially diffused into the pressboard, the conductivity in the upper layers is only seen by the longer wavelengths. The two 
competing factors equalize at time $t \approx 3 \mathrm{~h}$.

To parallel the data representation shown in Figure 12, the conductance plots are normalized to their final value (since the starting value is zero). Figure 14 shows that the results of the conductance normalization are very similar to the results of the capacitance normalization, including the reversal of the curve sequence between cases (a) and (b). The difference between the curves for individual wavelengths within the same top plate case is slightly less pronounced for the conductance, primarily because the growth over several orders of magnitude has been normalized to the linear $[0 ; 1]$ interval. Logarithmic representation of such data will be explored separately.

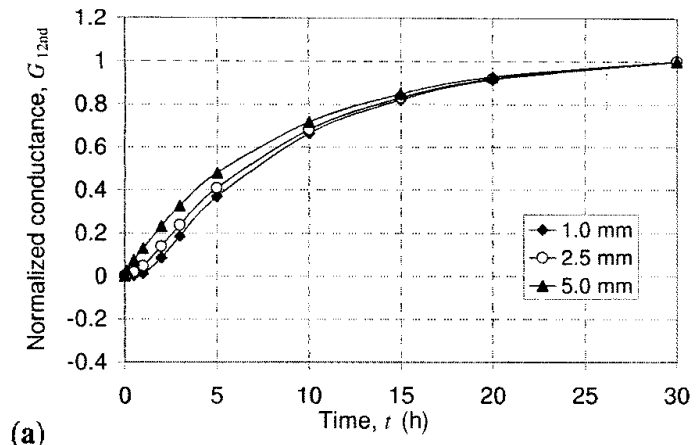

(a)

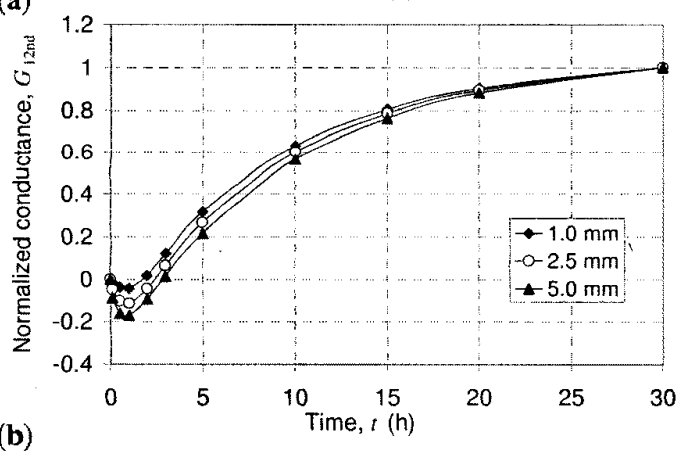

Figure 14. Change of conductance during the diffusion process with normalization to the final value for each of the three wavelengths, $G_{12 n d}=G_{12}(t) / G_{12}(t=\infty)$. (a) Without metal top plate, (b) with metal top plate:

\section{INVERSE PROBLEM}

\subsection{ASSUMPTION OF LINEARITY}

The simplest type of mapping from the interdigital sensor output usually assumes a linear relationship between the sensor capacitance, conductance, impedance, admittance, or resistance and the physical variable of interest (é.g. [47-49]). The advantages of such an approach include its inherent simplicity, stability, and speed. The disadvantages are numerous. Only relatively simple cases of spatial property distributions can be treated; the physics and chemistry of underlying phenomena are not fully represented; a posteriori information about the signal and frequent recalibration may be necessary; mass transfer in the bulk of material is reflected in the signal hysteresis.

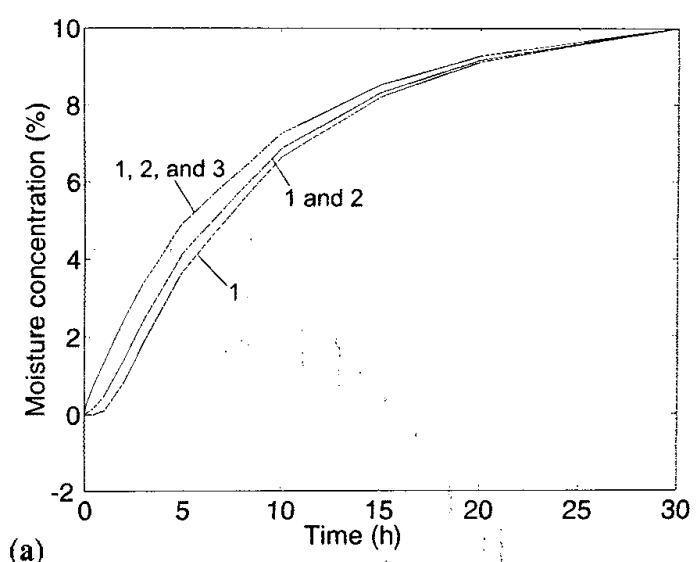

(a)

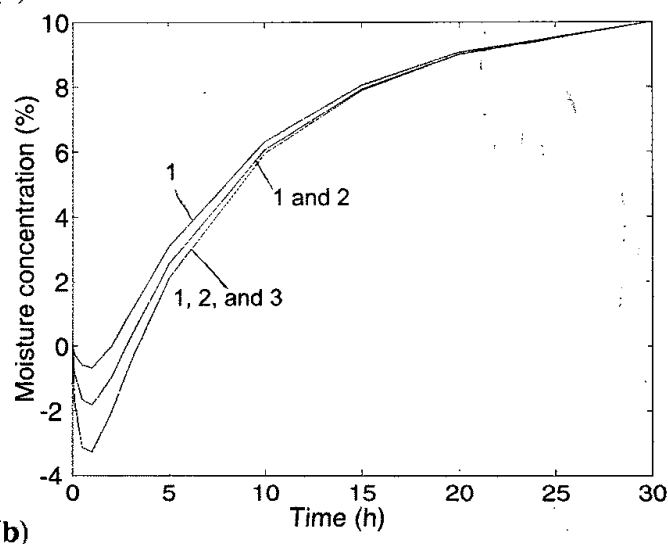

(b)

Figure 15. Time profiles of average moisture concentration calculated from simulated capacitance curves in Figure 12. The numbers next to the curves indicate the regions shown in Figure 4 penetrated with a corresponding spatial wavelength. The calculated moisture in part (b) is not physical at early time because of the negative moisture concentration values. (a) Without metal top plate, (b) with metal top plate.

The assumption of linearity for the case analyzed here is reasonable for relatively small variations for moisture concentration, and when the outer boundary moisture concentration is available from auxiliary sensor measurements. Bringing a perfect conductor in the vicinity of the sensor head significantly distorts the otherwise adequate linear relationship between moisture concentration and capacitance $C_{12}$ as was illustrated in Figures 10 through 14. Figure 15 shows the time variation of average moisture concentration detected by different electrode pairs. These profiles have been generated by a linear mapping of time profiles of the normalized capacitance $C_{12 n d}$ in Figure 12 from the $[0 ; 1]$ interval to moisture concentration in the interval $[0 ; 10 \%]$. In the field measurement, the upper limit of moisture concentration can be provided by an auxiliary moisture sensor immersed in transformer oil, using oil-pressboard equilibrium curves.

Figure 15 shows that such a mapping performs adequately for case (a), but produces negative, non-physical values of moisture concentration in case (b). Also, the sequence of curves in case (b) does not 
reflect average moisture concentrations in each layer of transformer pressboard because initially the normalized values decrease below the starting value. For that reason, only case (a) is analyzed from this point. By the nature of the mapping, the initial $(t=0)$ and final $(t=30 \mathrm{~h})$ moisture concentration profiles are guaranteed to be flat and assume extreme values of the moisture concentration interval $[0 ; 10] \%$.

\subsection{STAIR-STEP PROFILES}

From three-wavelength computer simulations, Figure 16 shows the calculated three-layer stair-step spatial profiles of moisture concentration at selected moments of time that directly correspond to the time profiles in Figure 15(a). Time (h) is indicated above each curve at each of the three layers of Figure 4 selected to represent pressboard thickness. The stair-step profiles provide intermediate, crude estimates of moisture concentration. For clarity of graphical presentation, the steps are plotted so that the level in the region 500 to $800 \mu \mathrm{m}$ shows the average level at the 500 to $1000 \mu \mathrm{m}$ region. Similarly, the level in the region 0 to $500 \mu \mathrm{m}$ shows the average level at the 0 to $1000 \mu \mathrm{m}$ region. The 800 to $1000 \mu \mathrm{m}$ region shows the average moisture level in the layer adjacent to the sensor. Appropriate weighting of these levels to reflect actual changes in each region is achieved in the next step by exploiting the outer boundary condition value at $x=0$. However, even at this point, the adequacy of the proposed approach is demonstrated as the moisture concentration decreases from left to right, as expected from the physical model.

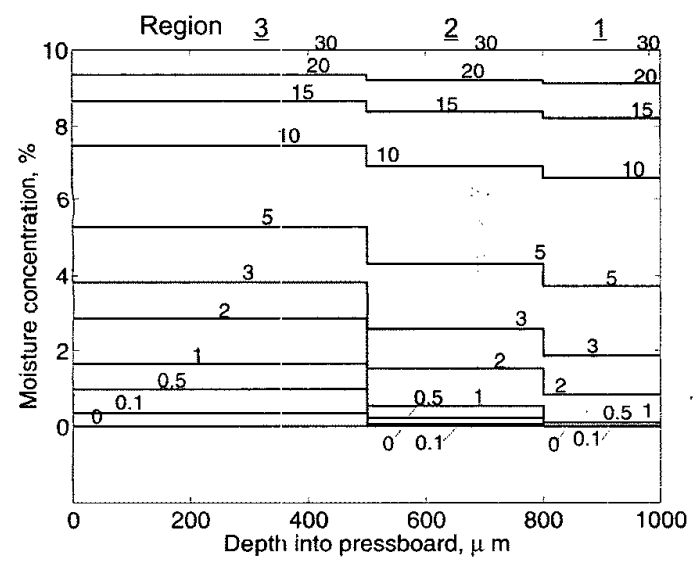

Figure 16. Stair-step profiles of average moisture concentration for selected moments of time plotted using the results in Figure 15(a), where the moisture values in Region 3 are obtained from curves $(1,2$, and 3), in Region 2 from curves (1 and 2), and in Region 1 from curve (1).

\subsection{CONTINUOUS PROFILES}

Additional information about the physical process is always valuable when solving inverse problems. Since the diffusion of moisture is being analyzed, we can impose a continuity requirement on each moisture profile. The discontinuities in Figure 16 are due to the spatial discretization of representation with the three-wavelength sensor shown in Figure 4. Also, the left boundary condition at $x=0$ may be available from auxiliary sensor measurements of oil moisture. In this benchmark problem, the left boundary condition is a constant moisture concentration of 10\%. Stair-step profiles in Figure 16 are first converted into point-wise profiles by assuming average concentration values at the region boundaries and a $10 \%$ concentration at the left boundary. Then the moisture profiles are approximated with a fourth-order polynomial in $x$. The result of this process is presented in Figure 17. The resultant profiles are close to the original ones shown in Figure 5 for all times except the very early ones. Very high gradients in the outer regions could not be characterized accurately due to the limited spatial resolution of the three-wavelength sensor.

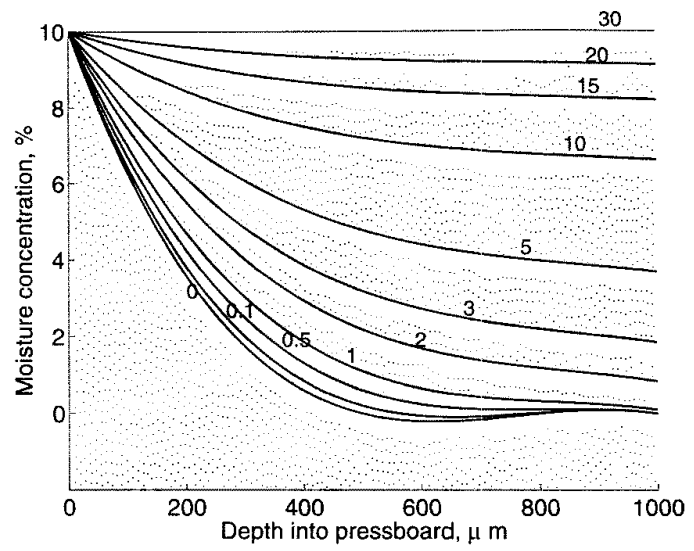

Figure 17. Smoothed continuous profiles of moisture concentration with an imposed $10 \%$ constant moisture concentration boundary condition at $x=0$ using a polynomial approximation of profiles in Figure 16. Profiles do not agree well with those of Figure 5 at early times $t<1 \mathrm{~h}$ because of the large gradients near $x=0$.

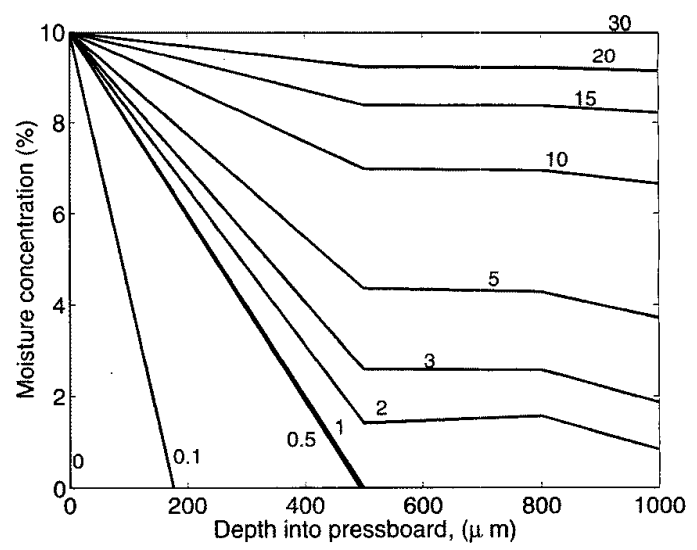

Figure 18. Piecewise-continuous moisture concentration profiles generated with imposed additional mass conservation requirement.

To address the steep gradients issue, one can incorporate a conservation of mass requirement while interpreting the stair-step parameter estimation provided by the three-wavelength sensor. It may be necessary to move the coordinates of the point that separates regions with dissimilar properties, which we will refer to as the collocation point. 
Figure 18 shows piecewise-continuous spatial profiles in which the collocation point departs from the region boundary and shifts to the left if the corresponding flat profile region in Figure 16 is at a very low moisture value. For example, the area of the triangle in Figure 18(a) at time $0.5 \mathrm{~h}$ is proportional to the area under the corresponding rectangle in Figure 16 that extends form $x=0$ to $x=500 \mu \mathrm{m}$. On the other hand, if the area of the triangle is larger than the rectangle area times the proportionality factor, the collocation point remains at the interlayer boundary. The proportionality factor should be selected based on the spatial rate of decay of the electric field in the material at increasing distances from the sensor head. In the first approximation chosen here, the triangle area has to be $5 \times$ as large as the rectangle area. The proportionality factor is somewhat dependent on material properties and has been selected empirically based on several simulations.

\section{EXPERIMENTAL RESULTS}

The results discussed in this Section, also presented in [50], are representative of many studies of moisture dynamics in transformer pressboard. The moisture concentration was measured in a $1.5 \mathrm{~mm}$ thick oilfree transformer pressboard as a function of time and position during the moisture diffusion process. A flexible interdigital three-wavelength sensor shown in Figure 2 was used to gather information regarding pressboard dielectric properties at different depths from the sensorpressboard interface. Data was obtained by processing the signals from the three-wavelength sensor, air humidity sensor, and thermocouple installed in the experimental chamber. Air-pressboard equilibrium curves were used to provide boundary conditions and the relative moisture level magnitude.

A controlled-environment experimental chamber was built to simulate the temperature, moisture, and materials of a power transformer environment [35]. A number of diffusion studies have been performed in this chamber to date. The ambient air inside the chamber was humidified to a pre-selected value by circulating moist air through a system of interconnected ducts.

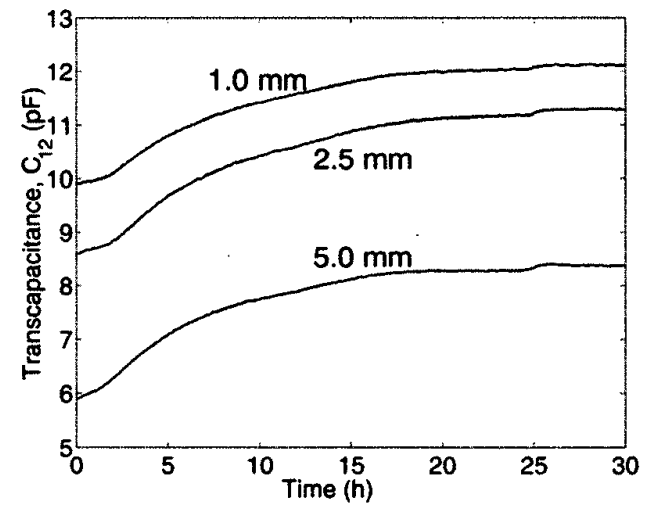

Figure 19. Measured capacitance between each electrode pair throughout the moisture diffusion experiment for each sensor wavelength at $1 \mathrm{~Hz}$.

Initially, the pressboard was vacuum-dried. The moisture diffusion process started with the step change in the ambient air humidity from
$0 \%$ to $\sim 12 \%$ with temperature being held at $70^{\circ} \mathrm{C}$. Air-pressboard equilibrium relationships [51] were used to provide boundary conditions at the air-pressboard interface and relative moisture level information needed for the parameter estimation algorithms. According to these relationships, a $12 \%$ humidity level in air at $70^{\circ} \mathrm{C}$ corresponds to $1.8 \%$ equilibrium moisture concentration in the transformer pressboard. Thus, the left side boundary condition at $x=0$ is a moisture level equal to $1.8 \%$ for the duration of the entire experiment.

The signals from the sensing electrodes of the three wavelengths were recorded at three frequencies: $0.1,1$, and $10 \mathrm{~Hz}$. This paper utilizes the signals only taken at the frequency of $1 \mathrm{~Hz}$, since the transcapacitance and transconductance were similar at 0.1 and $10 \mathrm{~Hz}$. Figure 19 shows the values of measured transcapacitance for each of the three electrode pairs. Each signal follows approximately the same pattern dictated by the diffusion dynamics. After a small initial delay, the signal grows at a high rate, after which it flattens off, indicating the end of the diffusion process experiment. The initial delay time is the smallest for the largest wavelength, because it can 'see' deepest into the pressboard.

Similar results hold for the measured transconductance vs. time for each of the three wavelengths. The relative amplitude of change of the transconductance is normally larger than that of the transcapacitance due to the high electric conductivity growth with the increase of the moisture concentration. Nevertheless, as demonstrated below, the transcapacitance provides sufficient sensitivity for the moisture profile measurements.

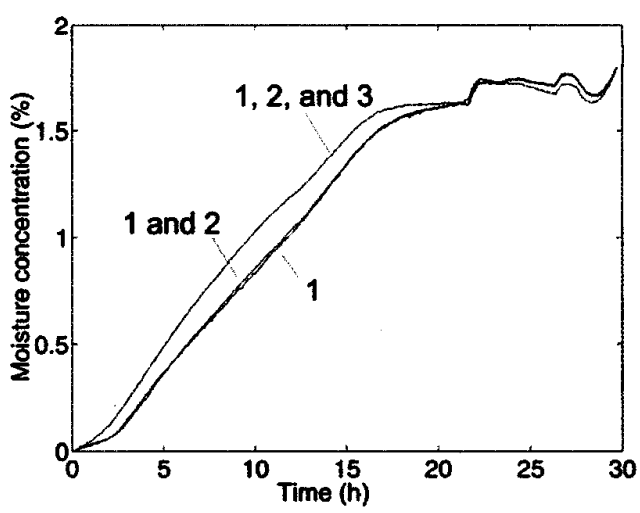

Figure 20. Average moisture level measured by each wavelength: region $1(1 \mathrm{~mm})$, regions 1 and $2(2.5 \mathrm{~mm})$, and regions 1,2 , and 3 $(5 \mathrm{~mm})$ using Figure 19 with (6).

\section{MOISTURE PROFILES}

The transcapacitance signals in Figure 19 were used to generate the curves of moisture concentration in the pressboard layers, assuming a linear relationship between the non-dimensional difference capacitance values of (5) and the moisture levels at each moment of time

$$
m(t)=m(t=0)+[m(t=\infty)-m(t=0)] C_{12 n d}(t)
$$

where for our experiment $m(t=0)=0$ and $m(t=\infty)=1.8 \%$. Note that conversion of measured capacitance values to moisture does not 
require any empirical relationship relating moisture to dielectric properties like those in (2) and (3).

Figure 20 shows the change in the average values of moisture concentration in the three regions that correspond to the penetration depths of the three wavelengths of the sensor. The average value corresponds to the cumulative amount of moisture in all regions within reach of a given penetration depth.

The fact that the difference of the moisture concentration measured by the 2.5 and the $1.0 \mathrm{~mm}$ wavelengths is very small, is related to a relatively small signal delay difference in the detection time analysis. Since the right side blocked boundary condition eliminates the moisture escape, the gradient-driven diffusion process results in a nearly flat moisture concentration profile across regions 1 and 2 of the pressboard indicated in Figure 4.

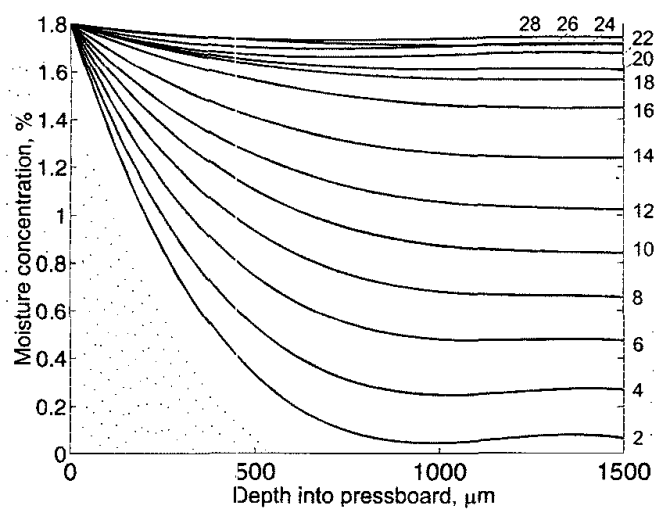

Figure 21. Calculated spatial profiles of moisture concentration across the thickness of pressboard from dielectrometry measurements using $D \approx 2.3 \times 10^{-11} \mathrm{~m}^{2} / \mathrm{s}$. Numbers next to the curves correspond to the time (h) at which the profile was measured. These curves were obtained using a polynomial approximation of the stair-step representation similar to that shown in Figure 16.

Figure 21 shows calculated moisture profiles from dielectrometry measurements for 14 distinct moments of time. The moisture spatial profiles were calculated from the measurement data using multivariable parameter estimation algorithms for the three-wavelength sensor combined with the moisture measurement data of the ambient environment (air relative humidity of $12 \%$ ) together with moisture equilibrium curves to give the pressboard moisture concentration of $1.8 \%$ at $x=0$. Early time profiles for $t<2 \mathrm{~h}$ are not shown because as earlier shown in the simulated analysis of Figure 17, the three wavelength sensor has insufficient resolution for high gradients near the $x=0$ interface.

The process starts with a step change in moisture concentration to $1.8 \%$ at the left boundary at time zero. The first two profiles (at the time moments of two hours and four hours) continue to show a slightly overestimated amount of moisture in the middle region of the pressboard because the sensor resolution is insufficient to capture high profile gradients. Future work will include better use of mass conservation requirements in order to improve the spatial resolution without adding more electrode pairs of different spatial periodicity. In this case, it may be possible to combine advantages of an approximate smooth profile representation and a straight line representation with moving collocation points.

The experiment is stopped at $\sim 30 \mathrm{~h}$, when the signals essentially stop changing. At this point, the moisture profile is expected to be almost uniform and at the maximum value of $1.8 \%$. The profile at $28 \mathrm{~h}$ has these characteristics.

Now, it is possible to evaluate the moisture diffusion coefficient from the moisture dynamics presented in Figure 21. By simulating the diffusion process using a standard finite difference technique, one can match the measured profiles. The estimated value of the diffusion coefficient is $D \approx 2.3 \times 10^{-11} \mathrm{~m}^{2} / \mathrm{s}$. Figure 22 shows theoretical moisture profiles generated using the estimated value of diffusion coefficient that are in reasonable agreement with the measured profiles in Figure 21.

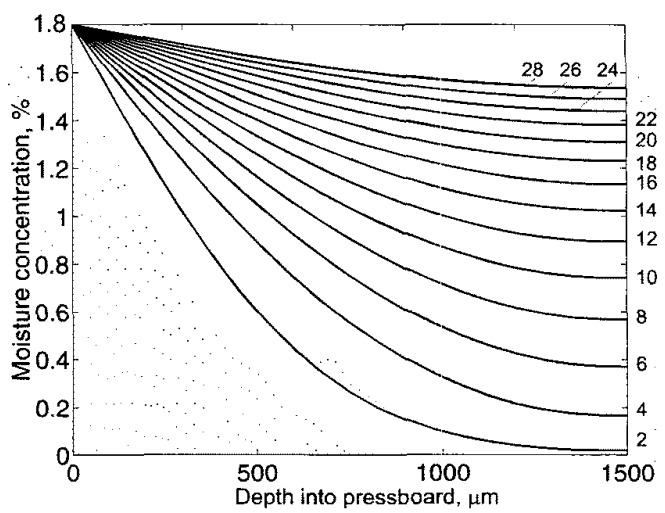

Figure 22. Theoretical spatial profiles of moisture concentration across the thickness of pressboard calculated using a diffusion coefficient of $D=2.3 \times 10^{-11} \mathrm{~m}^{2} / \mathrm{s}$, selected based on best visual match of the curves in the low gradient region. Numbers next to the curves correspond to the time (h) after the moisture was stepped to $1.8 \%$ at $x=0$.

\section{CONCLUSIONS AND FUTURE WORK}

$\mathrm{M}$ EASUREMENT of moisture diffusion processes in transformer pressboard have been made possible using multi-wavelength dielectrometry. The parameter estimation algorithm based on terminal characteristics of interdigital comb electrodes has been developed and tested against synthetic data. As a part of the algorithm testing process, the solution to a benchmark problem of a transient diffusion process has been calculated using finite element (FE) software. The benchmark problem will be used in the future to compare the performance of alternative parameter estimation algorithms. It also helps to develop intuition for analysis of multi-wavelength dielectrometry measurements and provides insights into improved experimental practices.

The algorithm tested here has demonstrated adequate performance for experimental setups which do not have sharp gradients of dielectric properties within the sensor penetration depth region of a wavelength. The algorithm has been successfully applied to experimental 
data. The moisture diffusion coefficient in oil-free transformer pressboard has been evaluated, assuming that it is independent of moisture concentration.

Future work includes testing of algorithms that determine spatially inhomogeneous dielectric properties as an intermediate step before evaluating the moisture concentration distribution. Measurements of moisture diffusion in oil-impregnated pressboard in a simulated transformer environment will be reported as well. The understanding of moisture-related processes in power transformers can be enhanced with this methodology. The results of measurements with oil-impregnated pressboard immersed in transformer oil will be published at a later date.

\section{ACKNOWLEDGMENT}

The authors would like to acknowledge the support of the Electric Power Research Institute, under grant WO 8619-01, managed by Mr. S. Lindgren, and the National Science Foundation under grant No. ECS-9523128. The donation of Maxwell software by Ansoft Corp. is gratefully appreciated. Financial support through APPA DEED scholarships to A. V. Mamishev and Y. Du and through a Link Foundation Fellowship to A. V. Mamishev is gratefully acknowledged. The authors would like to thank MIT undergraduate student Andrew Takahashi for assistance with data processing done as a part of the MIT Undergraduate Research Opportunities Program and an NSF REU grant. The work of J. H. Bau has also been a part of the MIT Undergraduate Research Opportunities Program.

\section{REFERENCES}

[1] P. A. von Guggenberg and M. C. Zaretsky, "Estimation of one-dimensional complexpermittivity profiles: a feasibility study", Journal of Electrostatics, Vol. 34, pp. 263277, Mar. 1995.

[2] V. K. Varadan, V. V. Varadan, and X. Q. Bao, "Integration of interdigital transducers, MEMS and antennas for smart structures", Smart Structures and Materials 1996 Smart Electronics and MEMS Conference, San Diego, CA, pp. 95-106, Feb. 1996.

[3] S. D. Senturia, N. F. Sheppard, Jr., H. L. Lee, and D. R. Day, "In-situ measurement of the properties of curing systems with microdielectrometry", Journal of Adhesion, Vol. 15, no. 69, pp. 69-90, 1982.

[4] N. T. Smith and D. D. Shepard, "Dielectric cure analysis: theory and industrial applications", Sensors, Vol. 12, pp. 42-48, Oct. 1995.

[5] E. S. Kolesar and J. M. Wiseman, "Interdigitated gate electrode field effect transistor for the selective detection of nitrogen dioxide and diisopropyl methylphosphonate", Analytical Chemistry, Vol. 61, pp. 2355-2361, 1989.

[6] J.W. Gardner, "Intelligent gas sensing using an integrated sensor pair", Sensors and Actuators B, Vol. 26-27, pp. 261-266, 1995.

[7] G. Niebling and A. Schlachter, "Qualitative and quantitative gas analysis with nonlinear interdigital sensor arrays and artificial neural networks", Sensors and Actuators B, Vol. B 26-27, pp. 289-292, 1995.

[8] A. Washabaugh, A. V. Mamishev, Y. Du, and M. Zahn, "Dielectric measurements of semi-insulating liquids and solids", International Conference on Conduction and Breakdown in Dielectric Liquids, Rome, Italy, pp. 381-384, July 1996.

[9] M. C. Zaretsky, J. R. Melcher, and C. M. Cooke, "Moisture sensing in transformer oil using thin-film microdielectrometry", IEEE Transactions on Electrical Insulation, Vol. 24, pp. 1167-1176, Dec. 1989.

[10] M. C. Zaretsky, P. Li, and J. R. Melcher, "Estimation of thickness, complex bulk permittivity and surface conductivity using interdigital dielectrometry", IEEE Transactions on Electrical Insulation, Vol. 24, pp. 1159-1166, Dec. 1989.

[11] J. O. Simpson and S. A. Bidstrup, "Modeling conductivity and viscosity changes during epoxy cure using TEA, DMA, and DSC", Proceedings of the American
Chemical Society, Division of Polymeric Materials: Science and Engineering, Fall Meeting, Vol. 69, no. 3, pp. 451-452, 1993.

[12] N. J. Goldfine, A. P. Washabaugh, J. V. Dearlove, and P. A. von Guggenberg, Imposed $\omega-k$ magnetometer and dielectrometer applications, Review of Progress in Quantitative Nondestructive Evaluation, D. Thompson and D. Chimenti, eds., Vol. 12, (New York), Plenum Press, 1993.

[13] Y. K. Sheiretov and M. Zahn, "Dielectrometry measurements of moisture dynamics in oil-impregnated pressboard", IEEE Transactions on Dielectrics and Electrical Insulation, Vol. 2, pp. 329-351, June 1995.

[14] N. F. Sheppard, D. R. Day, H. L. Lee, and S. D. Senturia, "Microdielectrometry", Sensors and Actuators, Vol. 2, pp. 263-274, July 1982.

[15] P. Li, Low Frequency, Millimeter Wavelength, Interdigital Dielectrometry of Insulating Media in a Transformer Environment. PhD thesis, Department of Electrical Engineering and Computer Science, Massachusetts Institute of Technology, Cambridge, MA, May 1987.

[16] M. C. Zaretsky, Parameter Estimation Using Microdielectrometry with Application to Transformer Monitoring. PhD thesis, Department of Electrical Engineering and Computer Science, Massachusetts Institute of Technology, Cambridge, MA, Nov. 1987.

[17] J. R. Melcher and M. C. Zaretsky, Apparatus and methods for measuring permittivity in materials, U.S. Patent No. 4,814,690, Mar. 21, 1989.

[18] Y. K. Sheiretov, Dielectrometry Measurements of Moisture Dynamics in Oil-Impregnated Pressboard, Master's thesis, Department of Electrical Engineering and Computer Science, Massachusetts Institute of Technology, Cambridge, MA, May 1994.

[19] Y. K. Sheiretov and M. Zahn, "Dielectrometry measurements of spatial moisture profiles in oil-impregnated pressboard", IEEE International Conference on Properties and Applications of Dielectric Materials, University of Queensland, Brisbane, Australia, pp. 701-704, July 1994.

[20] A. V. Mamishev, B. C. Lesieutre, and M. Zahn, "Optimization of multi-wavelength interdigital dielectrometry instrumentation and algorithms", IEEE Transactions on Dielectrics and Electrical Insulation, Vol. 5, pp. 408-420, 1998

[21] M. C. Zaretsky, L. Mouayad, and J. R. Melcher, "Continuum properties from interdigital electrode dielectrometry", IEEE Transactions on Electrical Insulation, Vol. 23, pp. 897-917, Dec. 1988.

[22] A. P. Washabaugh, P. A. von Guggenberg, M. Zahn, and J. R. Melcher, "Temperature and moisture transient flow electrification measurements of transformer pressboard/oil insulation using a Couette facility", Proceedings of The 3rd International Conference on Properties and Applications of Dielectric Materials, Vol. 2, Tokyo, Japan, pp. 867-870, July 8-12, 1991.

[23] A. J. Morin, M. Zahn, and J. R. Melcher, "Fluid electrification measurements of transformer pressboard/oil insulation in a Couette charger", IEEE Transactions on Electrical Insulation, Vol. 26, pp. 870-901, Oct. 1991.

[24] W. L. Teague and J. H. McWhirter, "Dielectric measurements on new power transformer insulation", Transactions of the American Institute of Electrical Engineers (AIEE), Part III: Power Apparatus and Systems, Vol. 71, pp. 743-752, Oct. 1952.

[25] R. T. Rushall, "Dielectric properties of oil-soaked pressboard as affected by water", Proceedings of The Institution of Electrical Engineers, Part IIA, Vol. 100, pp. 81-88, Mar. 1953.

[26] A. W. Stannett, "The measurement of water in power transformers", Proceedings of The Institution of Electrical Engineers, Part A: Power Engineering, Supplement No. 3, Vol. 109, pp. 80-85, June 1962.

[27] T. E. Constandinou, "Relation between moisture content and low-voltage electrical properties of oil-impregnated, resin-coated and unimpregnated papers", Proceedings of The Institution of Electrical Engineers, Vol. 112, pp. 1783-1794, Sept. 1965.

[28] I. Gussenbauer, "Examination of humidity distribution in transformers models by means of dielectric measurements", Proceedings of the International Conference on Large High Voltage Electric Systems (CIGRÉ), Vol. I, (Paris, France), Aug. 27-Sept. 4, 1980. Paper No. 135.

[29] Y. Saito and T. Hino, "A study of thermal deterioration of Kraft pulps using a mass spectrometer", Transactions of the American Institute of Electrical Engineers (AIEE), Part I: Communication and Electronics, Vol. 78, pp. 602-606, Nov. 1959.

[30] J. Fabre and A. Pichon, "Deterioration processes and products of paper and oil. Application to transformers", Proceedings of the International Conference on Large High Voltage Electric Systems (CIGRÉ), (Paris, France), June 15-25, 1960. Paper No. 137 . 
[31] D. H. Shroff and A. W. Starnett, "A review of paper aging in power transformers", IEE Proceedings, Part C: Generation, Transmission and Distribution, Vol. 132, pp. 312-319, Nov. 1985.

[32] F. M. Clark, "Factors affecting the mechanical deterioration of cellulose insulation", Transactions of Electrical Engineering, Vol. 61, pp. 742-749, Oct. 1942.

[33] H. P. Moser, V. Dahinden, H. Friederich, H. Künast, K. Lennarz, U. Leukens and O. Potocnik, Transformerboard. St:. Johnsbury, Vermont: Scientia Electrica, special publication, 1979.

[34] H. P. Moser and V. Dahinden, Transformerboard II. Rapperswil, Switzerland: H. Weidmann $A G, 1987$.

[35] Y. Du, B. C. Lesieutre, and M. Zahn, "Dielectrometry measurements of effects of moisture and anti-static additive on transformer board", IEEE Conference on Electrical Insulation and Dielectric Phenomena, Minneapolis, MN, pp. 226-229, Oct. 1997.

[36] A. V. Mamishev, Y. Du, B. C. Lesieutre, and M. Zahn, "Measurement of stratified distributions of dielectric properties and dependent physical parameters. Graduate student award finalist paper", Fall Meeting of Materials Research Society, Boston, MA, pp. 29-34, Dec. 1997.

[37] R. S. Jachowicz and S. D. Senturia, "A thin-film capacitance humidity sensor", Sensors and Actuators, Vol. 2, pp. 171-186, Dec. 1981.

[38] M. Hijikigawa, T. Sugihara, J. Tanaka, and M. Watanabe, "Micro-chip FET humidity sensor with a long-term stability", Transducers '85. 1985 International Conference on Solid-State Sensors and Actuators, Philadelphia, PA, pp. 221-224, June 1985.

[39] D. R. Day and D. D. Shepard, "Dynamic cure and diffusion monitoring in thin encapsulant films", Nondestructive Monitoring of Materials Properties Symposium, J. Holbrook and J. Bussiere, eds., Boston, MA, pp. 227-232, Nov. 1988.

[40] T. Lang, H.-D. Wiemhöfer, and W. Göpel, "Carbonate based $\mathrm{CO}_{2}$ sensors with high performance", Sensors and Actuators B, Vol. B34, pp. 382-387, Jan. 1996.

[41] H. E. Endres, S. Drost, and F! Hunter, "Impedance spectroscopy on dielectric gas sensors", Sensors and Actuators B, Vol. 22, pp. 7-11, 1994.
[42] R. F. Taylor, I. G. Marenchic, and E. J. Cook, "An acetylcholine receptor-based biosensor for the detection of cholinergic agents", Analytica Chimica Acta, Vol. 213, pp. $131-138,1988$

[43] D. C. Cullen, R. S. Sethi, and C. R. Lowe, "Multi-analyte miniature conductance biosensor", Analytica Chimica Acta, Vol. 231, pp. 33-40, 1990.

[44] U. Gäfvert, A. Jaksts, C. Tornkvist, and L. Walfridsson, "Electric field distribution in transformer oil", IEEE Transactions on Electrical Insulation, Vol. 27, pp. 647-660, June 1992.

[45] B. Nettelblad, "Effect of moisture content on the dielectric properties of cellulose", NORD-IS-92, 1992.

[46] A. K. Jonscher, Dielectric Relaxation in Solids. Chelsea Dielectrics Press, London, 1983.

[47] G. Huyberechts and L. Frisson, "In situ formation of humidity-sensitive devices for the evaluation of solar panel encapsulations", Sensors and Actuators B, Vol. 26-27, pp. 308-311, 1995

[48] K. D. Schierbaum, J. Geiger, U. Weimar, and W. Göpel, "Specific palladium and platinum doping for $\mathrm{SnO}_{2}$-based thin film sensor arrays", Sensors and Actuators B, Vol. 13-14, pp. 143-147, 1993.

[49] J. M. Fouke, A. D. Wolin, K. G. Sanders, M. R. Neuman, and E. R. McFadden, Jr., "Sensor for measuring surface fluid conductivity in vivo", IEEE Transactions on Biomedical Engineering, Vol. 35, pp. 877-881, Oct. 1988.

[50] A. V. Mamishev, Y. Du, B. C. Lesieutre, and M. Zahn, "Measurement of moisture spatial profiles in transformer pressboard", IEEE Conference on Electrical Insulation and Dielectric Phenomena, Atlanta, GA, pp. 323-326, Oct. 1998.

[51] R. Jeffries, "The sorption of water by cellulose and eight other textile polymers", Journal of the Textile Institute, Vol. 51, no. 9, pp. 339-374, 1960.

This paper is based on a presentation given at the 13 th International Conference on Dielectric Liquids, Nara, Japan, 20-25 July 1999.

Manuscript was received on 22 October 1999. 